\title{
Relationship Between Respiratory Muscle Strength and Conventional Sarcopenic Indices in Young Adults: A Preliminary Study
}

\author{
Hee Joon Ro, MD, Don-Kyu Kim, MD, PhD, Sang Yoon Lee, MD, \\ Kyung Mook Seo, MD, PhD, Si Hyun Kang, MD, PhD, Hoon Chang Suh, MD
}

Department of Physical Medicine and Rehabilitation, Chung-Ang University College of Medicine, Seoul, Korea

Objective To investigate the relationships between respiratory muscle strength and conventional sarcopenic indices such as skeletal muscle mass and limb muscle strength.

Methods Eighty-nine young adult volunteers who had no history of medical or musculoskeletal disease were enrolled. Skeletal muscle mass was measured by bioelectrical impedance analysis and expressed as a skeletal muscle mass index (SMI). Upper and lower limb muscle strength were evaluated by hand grip strength (HGS) and isometric knee extensor muscle strength, respectively. Peak expiratory flow (PEF), maximal inspiratory pressure (MIP), and maximal expiratory pressure (MEP) were evaluated using a spirometer to demonstrate respiratory muscle strength. The relationships between respiratory muscle strength and sarcopenic indices were investigated using Pearson correlation coefficients and multiple linear regression analysis adjusted by age, height, and body mass index.

Results MIP showed positive correlations with SMI ( $\mathrm{r}=0.457$ in men, $\mathrm{r}=0.646$ in women; both $\mathrm{p}<0.01$ ). MIP also correlated with knee extensor strength ( $\mathrm{p}<0.01$ in both sexes) and HGS ( $\mathrm{p}<0.05$ in men, $\mathrm{p}<0.01$ in women). However, PEF and MEP had no significant correlations with these sarcopenic variables. In multivariate regression analysis, MIP was the only independent factor related to SMI $(\mathrm{p}<0.01)$.

Conclusion Among the respiratory muscle strength variables, MIP was the only value associated with skeletal muscle mass.

Keywords Sarcopenia, Respiratory muscles, Muscle strength, Skeletal muscle, Spirometry

Received April 17, 2015; Accepted June 8, 2015

Corresponding author: Don-Kyu Kim

Departments of Physical Medicine and Rehabilitation, Chung-Ang University College of Medicine, 102 Heukseok-ro, Dongjak-gu, Seoul 06973, Korea Tel: +82-2-6299-1881, Fax: +82-2-6298-1866, E-mail: donkim21@cau.ac.kr

() This is an open-access article distributed under the terms of the Creative Commons Attribution Non-Commercial License (http://creativecommons.org/ licenses/by-nc/4.0) which permits unrestricted noncommercial use, distribution, and reproduction in any medium, provided the original work is properly cited. Copyright $\odot 2015$ by Korean Academy of Rehabilitation Medicine 


\section{INTRODUCTION}

Sarcopenia is the age-related loss of skeletal muscle mass, muscle strength, and physical performance [1]. Since one longitudinal study suggested cut-off values of skeletal muscle mass for defining sarcopenia [2], a number of studies have been performed to suggest a specific definition of sarcopenia. The European Working Group on Sarcopenia in Older People (EWGSOP) used low muscle function (muscle strength or physical performance) accompanied by low skeletal muscle mass to diagnose sarcopenia [3]. Although dynapenia, which is defined as the age-related loss of muscle strength, was considered to result from the loss of skeletal muscle mass [4], a recent study has suggested that the decrease in muscle strength is significantly more rapid than the concomitant loss of muscle mass [5]. Moreover, one study also reported that maintaining or even gaining muscle mass does not prevent age-related decreases in muscle strength [5].

The EWGSOP used knee flexor/extensor strength, hand grip strength (HGS), and peak expiratory flow (PEF) to measure muscle strength [3]. Knee flexor/extensor strength is known as a direct and important indicator of gait and physical function in the elderly population $[6,7]$. Isokinetic dynamometers are considered to be reliable and objective for measuring lower extremity muscle strength [8]. However, they are expensive, not portable, occupy space, and require long measurement times and trained raters [9]. A hand-held dynamometer can measure lower extremity muscle strength, but it has multiple limitations in its measurement position, joint angle, measurement site and type, and muscle contraction type and speed $[10,11]$. HGS is also recommended as a good simple measure of muscle strength according to the diagnostic algorithm of the EWGSOP [3]. Recently, the Asia Working Group for Sarcopenia proposed that HGS measurement is a feasible and convenient measure of muscle strength because of cost, availability, and ease of use [12]. Although HGS is convenient to measure, it has limitations in elderly people with arthritis of the wrist or hand and reflects only the localized function of the upper extremity.

However, sarcopenia is not confined to only upper or lower limb muscles but involves generalized loss of skeletal muscle mass and strength. Sarcopenia may also affect respiratory muscles and decrease functional capacity, affecting activities of daily living. It has been suggested that sarcopenia initiates a chain of events that lead to reduced pulmonary function and low physical performance $[13,14]$. Thus, to minimize respiratory complications, it is important to understand how sarcopenia changes respiratory function. The EWGSOP used PEF to measure respiratory muscle strength. However, research on the use of PEF as a measure of sarcopenia is limited, so it cannot be recommended as an isolated measure of muscle strength at this time [3].

Generally, maximum inspiratory and expiratory pressure are simple to measure and are helpful indicators of respiratory muscle weakness [15]. Clinically, maximum inspiratory pressure (MIP) is a widely applied technique for assessing inspiratory muscle strength. Maximum expiratory pressure (MEP) is the only generally available test for measuring expiratory muscle power [16]. Maximal respiratory pressure is disturbed early and is the single most sensitive measure of respiratory muscle weakness. They are measured using general tests of the combined neuromuscular function of the diaphragm, abdominal, intercostal, and accessory muscles [15]. To our knowledge, there is no report about the relationship between skeletal muscle mass and these respiratory muscle strength variables, although MIP and MEP are affected by sarcopenia.

Although sarcopenia is a phenomenon of aging, in a study about respiratory muscles in elderly people, it is complicated to control for a number of variables, such as many respiratory diseases including chronic obstructive pulmonary disease. Thus, we planned a preliminary study that targeted healthy young adults who had no respiratory diseases. In this preliminary study, we investigated the relationships between respiratory muscle strength and conventional sarcopenic indices in healthy young adults.

\section{MATERIALS AND METHODS}

\section{Participants}

A total of 89 healthy young adult volunteers (53 men and 36 women) ages 20 to 39 were enrolled. Participants who had musculoskeletal diseases at the time of their recruitment, a history of major joint surgery that could have affected their limbs, abnormal physical examination findings in their joints, or a history of chronic medical ill- 
nesses such as hypertension, diabetes, or thyroid disease were excluded from this study. The study protocol was approved by the Institutional Review Board of ChungAng University Hospital (C2013236 [1196]). The subjects were recruited through advertisements and informed of the purpose and nature of this study on the written consent form.

\section{Skeletal muscle mass assessment}

Bioelectrical impedance analysis (InBody S10; Biospace, Seoul, Korea) was used to analyze the participants' body composition. Measuring the skeletal muscle mass using this instrument was already demonstrated to be effective in a number of clinical studies $[17,18]$. To improve test reliability, participants refrained from eating or exercising for at least 8 hours prior to the measurements and were instructed to urinate immediately before the measurements were taken. After height and weight were measured, four electrodes were attached to the extremities of each participant in the supine position [19]. As an index of muscle mass, we used the skeletal muscle mass index (SMI) calculated as [20]:

$$
\text { SMI }(\%)=\text { Skeletal muscle mass }(\mathrm{kg}) / \text { Weight }(\mathrm{kg}) \times 100
$$

\section{Limb muscle strength measurements}

HGS was measured using a hand-grip dynamometer (T.K.K.5401; Takei Scientific Instruments, Tokyo, Japan) that was widely used in clinical studies [21,22]. Participants were asked to assume the following position while sitting in a straight-backed chair with the feet flat on the floor: adduct and neutrally rotate the shoulder, flex the elbow to $90^{\circ}$, and place the forearm in a neutral position, with the wrist between $0^{\circ}$ and $30^{\circ}$ extension and between $0^{\circ}$ and $15^{\circ}$ ulnar deviation. The arm was not supported by the examiner or by an armrest. For grip strength measurement, a hand-grip dynamometer was presented in line with the forearm. Participants were instructed to squeeze the handle as hard as possible for 3 seconds, and the maximum contraction force $(\mathrm{N})$ was recorded. The tests were performed three times for both hands, alternating between the right and left hands with a 60 -second rest between each trial [23]. The highest value among the three repeated measurements was used for the analysis.

Knee extensor muscle strength (KES) was tested with a hand-held dynamometer (JTECH Medical, Salt Lake
City, UT, USA). KES assessment using this instrument was revealed to be reliable and valid in one study [24]. The participants sat upright on a table with both lower legs hanging down, with the knees flexed at $90^{\circ}$ and the thighs in a horizontal position. The examiner placed the hand-held dynamometer two fingerbreadths above the ankle joint and distal to the tibia and held it in place with an ankle belt. The participants were requested to push as hard as they could against the examiner's hand while the examiner pushed back until the examiner could break the position by overpowering the participants' isometric exertion [25]. Maximal knee extension force (N) was measured three times for each leg. The measurement was performed at greater than 30-second intervals. To calculate the peak torque $(\mathrm{Nm})$, the distance $(\mathrm{m})$ between the knee joint and the ankle was measured and then multiplied by the muscle force $(\mathrm{N})$ measured by the handheld dynamometer. The highest value was selected as the maximal knee extensor strength and was used for the analysis. In order to prevent muscle fatigue, each muscle strength test was conducted at five-minute intervals.

\section{Respiratory muscle strength measurements}

Respiratory muscle strength was assessed by measuring PEF, MIP, and MEP using a spirometer (COSMED, Rome, Italy) that was widely used in clinical studies $[26,27]$. Spirometry was carried out in the sitting position while the participant wore a nose clip. Participants were instructed to prevent air leaks around the mouthpiece by pinching their lips around it and to support their cheeks during their expiratory efforts. To measure PEF, the examiner instructed the participants to exhale as quickly as possible through the mouthpiece after maximum inspiration and then repeat the maneuver three times. To assess MIP and MEP, subjects maintained maximal pressure in the same position for at least 2 seconds after inhaling or exhaling. MIP was measured at residual volume, and MEP was measured at total lung capacity. The participants carried out at least three acceptable inspirations/expirations to determine reproducible inspirations/expirations. The maximum values of each maneuver that varied by less than $20 \%$ were recorded [28].

\section{Statistical analysis}

We analyzed the skeletal muscle mass and various muscle strength measurements classified by sex because 
Table 1. Characteristics of subjects according to sex

\begin{tabular}{|lccc}
\hline & Men $(\mathbf{n}=\mathbf{5 3})$ & Women $(\mathbf{n}=\mathbf{3 6})$ & p-value \\
\hline Age $(\mathrm{yr})$ & $26.5 \pm 3.6$ & $26.7 \pm 3.4$ & 0.722 \\
\hline Height $(\mathrm{cm})$ & $176.37 \pm 4.85$ & $162.47 \pm 4.95$ & $<0.001^{*}$ \\
\hline Body mass index $\left(\mathrm{kg} / \mathrm{m}^{2}\right)$ & $23.7 \pm 2.5$ & $20.4 \pm 1.7$ & $<0.001^{*}$ \\
Skeletal muscle mass index $(\%)$ & $46.35 \pm 4.07$ & $41.44 \pm 2.48$ & $<0.001^{*}$ \\
Peak expiratory flow $(\mathrm{L} / \mathrm{s})$ & $10.1 \pm 1.5$ & $7.0 \pm 1.1$ & $<0.001^{*}$ \\
MIP $\left(\mathrm{cmH}_{2} \mathrm{O}\right)$ & $82.4 \pm 16.9$ & $64.7 \pm 12.1$ & $<0.001^{*}$ \\
MEP $\left(\mathrm{cmH}_{2} \mathrm{O}\right)$ & $83.1 \pm 18.2$ & $62.8 \pm 11.6$ & $<0.001^{*}$ \\
KES PT $(\mathrm{Nm})$ & $182.91 \pm 16.68$ & $111.99 \pm 12.82$ & $<0.001^{*}$ \\
\hline Handgrip strength $(\mathrm{N})$ & $44.8 \pm 4.4$ & $27.9 \pm 3.8$ & $<0.001^{*}$ \\
\hline
\end{tabular}

Values are presented as mean \pm standard deviation.

MIP, maximal inspiratory pressure; MEP, maximal expiratory pressure; KES, knee extensor strength; PT, peak torque.

${ }^{*} \mathrm{p}<0.001$ by independent $\mathrm{t}$-test.

muscle strength differs between men and women. The demographic characteristics and laboratory variables of the participants according to sex were compared using an independent t-test. Correlations between SMI and the muscle strength variables were analyzed using Pearson correlation coefficient. Multiple linear regression analysis was used to determine the independent effect of each respiratory muscle strength parameter on SMI adjusted for age, height, body mass index (BMI), and limb muscle strength. For statistical analysis, SPSS ver. 17.0 (SPSS Inc., Chicago, IL, USA) was used. The results were considered to be statistically significant at $\mathrm{p}<0.05$.

\section{RESULTS}

\section{Participant characteristics}

Characteristics of the study population are shown in Table 1. The mean age of the subjects was $26.56 \pm 3.48$ years. The participants' mean SMI was $46.35 \% \pm 4.07 \%$ in men and $41.44 \% \pm 2.48 \%$ in women. The mean respiratory muscle strength values assessed by PEF, MIP, and MEP in men were significantly larger than those in women $(10.14 \pm 1.45$ vs. $7.01 \pm 1.07 \mathrm{~L} / \mathrm{s}, 82.40 \pm 16.87$ vs. $64.69 \pm 12.13$ $\mathrm{cmH}_{2} \mathrm{O}$, and $83.11 \pm 18.19$ vs. $62.75 \pm 11.60 \mathrm{cmH}_{2} \mathrm{O}$, respectively; $\mathrm{p}<0.001$ in all). Men also showed greater limb muscle strength assessed by KES and HGS than did women $(\mathrm{p}<0.001$ in all).

Correlation between respiratory muscle strength and muscle mass

In the correlation between SMI and respiratory muscle
Table 2. Pearson correlation coefficients (r) among respiratory muscle strength, limb strength, and skeletal muscle mass

\begin{tabular}{lllllllll}
\hline & \multicolumn{3}{c}{ Men } & & \multicolumn{3}{c}{ Women } \\
\cline { 2 - 4 } \cline { 6 - 8 } & SMI & KES & HGS & & SMI & KES & HGS \\
\hline PEF & 0.112 & $0.279^{*}$ & 0.169 & & 0.107 & 0.150 & 0.257 \\
MIP & $0.457^{* *}$ & $0.428^{* *}$ & $0.306^{*}$ & & $0.646^{* *}$ & $0.469^{* *}$ & $0.611^{* *}$ \\
MEP & 0.268 & $0.376^{* *}$ & 0.255 & & 0.328 & 0.183 & 0.183 \\
\hline
\end{tabular}

SMI, skeletal muscle mass index; KES, knee extensor strength; HGS, hand grip strength; PEF, peak expiratory flow; MIP, maximal inspiratory pressure; MEP, maximal expiratory pressure.

${ }^{*} \mathrm{p}<0.05,{ }^{* *} \mathrm{p}<0.01$.

strength, only MIP correlated strongly with SMI in men and women. The correlation coefficients between MIP and SMI were 0.457 (men) and 0.646 (women) ( $\mathrm{p}<0.01$ in both sexes). However, PEF and MEP were not significantly associated with SMI in either sex (Table 2, Fig. 1).

Correlation between respiratory muscle strength and limb muscle strength

In the correlation between respiratory and limb muscle strength, only MIP showed a significant correlation with KES and HGS, with the correlation coefficients being $0.428(\mathrm{p}<0.01)$ and $0.306(\mathrm{p}<0.05)$ in men and 0.469 $(\mathrm{p}<0.01)$ and $0.611(\mathrm{p}<0.01)$ in women. However, PEF and MEP were only associated significantly with KES in men and not associated with limb muscle strength in any area in women (Table 2). 
(A)

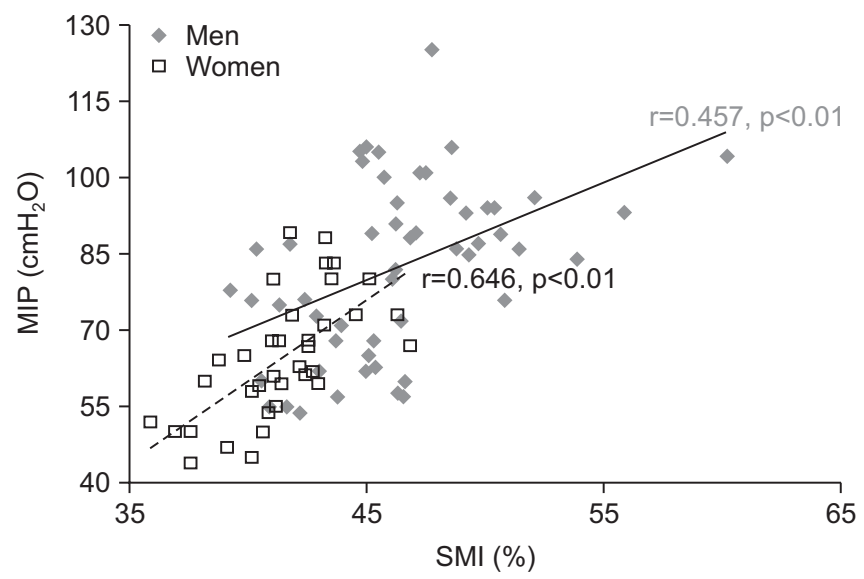

(B)

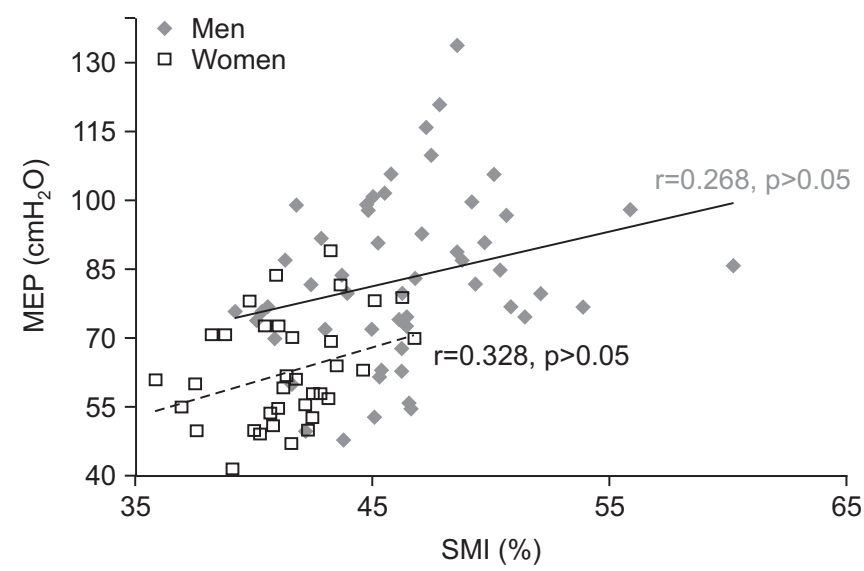

Fig. 1. Correlation is shown between (A) maximal inspiratory pressure (MIP) and (B) maximal expiratory pressure (MEP) with skeletal muscle mass. The blue diamonds and solid lines show the results for men; the red hollow squares and dotted lines represent the results for women. SMI, skeletal muscle mass index.

Table 3. Multiple linear regression analysis for various parameters including muscle strength associated with skeletal muscle mass index

\begin{tabular}{|c|c|c|c|c|c|c|}
\hline & \multicolumn{3}{|c|}{ Men } & \multicolumn{3}{|c|}{ Women } \\
\hline & \multirow{2}{*}{$\beta$} & \multicolumn{2}{|c|}{ 95\% CI } & \multirow{2}{*}{$\beta$} & \multicolumn{2}{|c|}{$95 \%$ CI } \\
\hline & & Lower & Upper & & Lower & Upper \\
\hline Age & -0.029 & -0.242 & 0.177 & -0.188 & -0.295 & 0.019 \\
\hline Height & -0.101 & -0.242 & 0.073 & -0.048 & -0.162 & 0.113 \\
\hline BMI & $-0.520^{* *}$ & -1.145 & -0.549 & $-0.498^{* *}$ & -1.017 & -0.442 \\
\hline PEF & 0.042 & -0.396 & 0.631 & 0.035 & -0.385 & 0.547 \\
\hline MIP & $0.401^{*}$ & 0.023 & 0.170 & $0.341^{*}$ & 0.001 & 0.139 \\
\hline MEP & -0.258 & -0.124 & 0.009 & 0.003 & -0.058 & 0.059 \\
\hline KES & $0.249^{*}$ & 0.006 & 0.116 & 0.048 & -0.048 & 0.067 \\
\hline HGS & $0.298^{* *}$ & 0.088 & 0.463 & $0.478^{* *}$ & 0.047 & 0.576 \\
\hline
\end{tabular}

BMI, body mass index; PEF, peak expiratory flow; MIP, maximal inspiratory pressure; MEP, maximal expiratory pressure; KES, knee extensor strength; HGS, hand grip strength; $\beta$, standardized coefficients.

${ }^{*} \mathrm{p}<0.05,{ }^{* *} \mathrm{p}<0.01$.

Multiple linear regression analysis of the relationship between skeletal muscle mass and respiratory muscle strength

In regression analysis that included age, height, BMI, PEF, MIP, MEP, KES, and HGS, factors related to SMI in men were BMI, MIP, KES, and HGS ( $\beta=-0.520$, 95\% CI -1.145 to $-0.549, p=0.001 ; \beta=0.401,95 \%$ CI 0.023 to 0.170 , $\mathrm{p}=0.011 ; \beta=0.249,95 \%$ CI 0.006 to $0.116, p=0.030 ; \beta=0.298$, $95 \%$ CI 0.088 to $0.463, p=0.005$, respectively), and those that were related to SMI in women were BMI, MIP, and HGS ( $\beta=-0.498,95 \%$ CI -1.017 to $-0.442, p=0.001 ; \beta=0.341$,
95\% CI 0.001 to $0.139, p=0.048 ; \beta=0.478,95 \%$ CI 0.047 to $0.576, p=0.023$, respectively) (Table 3 ).

\section{DISCUSSION}

In this preliminary study of healthy young adults, we measured muscle mass, upper and lower extremity muscle strength, and respiratory muscle strength. We found that among the respiratory muscle strength parameters, only MIP correlated with muscle mass. We also found that muscle mass and MIP were independently correlated 
after we adjusted for age, height, BMI, and limb muscle strength in both men and women. To our knowledge, this is the first study to reveal the independent relationship between skeletal muscle mass and respiratory muscle strength.

Sarcopenia has been mostly investigated with respect to limb muscle mass and function rather than respiratory muscle function. Numerous studies have reported that decreased limb muscle strength is associated with decreased physical performance [6,7], physical disability $[29,30]$, and even death [31] in older persons. However, the etiology of decreased physical function or death could be associated directly with sarcopenia of the respiratory muscles $[13,14,32]$. Recently, sarcopenia of the diaphragm in aging mice was demonstrated, and this change in the diaphragm may contribute to respiratory complications with aging [33]. However, this finding is limited only to the effect of the diaphragm on pulmonary function. Weakness of the respiratory muscles is an important problem that leads to a number of pulmonary complications and death. In one study, reduced MIP was an independent risk factor for cardiovascular events such as myocardial infarction, cardiovascular disease, stroke, and death [34]. Huang et al. [35] reported that inspiratory muscle strength training increases MIP and improves functional status in older adults without chronic obstructive pulmonary disease. Thus, assessing respiratory muscle strength is invaluable for estimating functional capacity and medical prognosis.

The EWGSOP recommended measuring PEF to determine respiratory muscle strength because it is an inexpensive, simple, and widely accessible technique. However, PEF is not an appropriate indicator of respiratory muscle strength because it evaluates the extent of airflow limitation. In other words, a change in PEF reflects alteration in the caliber of large airways [36]. Thus, PEF monitoring has been used for clinically managing patients with asthma. Although one study reported that expiratory muscle strength training could affect the cough function in the elderly, which is correlated with PEF [37], another study showed a contradictory result. Tamplin and Berlowitz [38] suggested that respiratory muscle training appears to be effective at increasing MIP and MEP, whereas no effect was found for PEF. Therefore, we thought that the validity of PEF in sarcopenic research could be deferred and that additional studies are needed. The most widely used tests of global respiratory muscle strength are the static maximum pressures (MIP and MEP) measured through the mouth [16]. MIP and MEP can easily be measured with a portable mouth pressure meter in the clinical setting [15]. Thus, MIP and MEP might be better sarcopenia parameters than PEF for assessing precise respiratory muscle strength.

A number of studies have addressed the relationships between respiratory strength and limb strength. Simoes et al. [39] reported that both MIP and MEP were positively correlated with knee flexor and extensor strength in the elderly. Buchman et al. [13] also suggested that upper and lower extremity muscle strength were correlated with respiratory muscle strength in 960 elderly patients. The current study also revealed positive correlations between respiratory muscle strength and limb muscle strength. However, in the current study, limb muscle strength was positively correlated only with MIP, not MEP, unlike the aforementioned studies.

Although MIP and MEP both reflect respiratory muscle strength, only MIP showed an independent association with the sarcopenic index in this study. This finding might originate from the differences between the inspiratory and expiratory muscles. The diaphragm, internal intercostals of the parasternal region, external intercostals, and other accessory muscles mainly constitute the inspiratory muscles. The lateral internal intercostals and abdominal muscles constitute the expiratory muscles [40]. Because the muscle mass of inspiration is greater than that of expiration, we postulate that MIP could reflect a person's sarcopenic status more accurately than MEP. To our knowledge, there is no report about the timing of inspiratory and expiratory muscle involvement with sarcopenia.

There are a number of limitations in this study. First, all participants were young adults. As a preliminary study to investigate the relationship between respiratory muscle strength, limb muscle strength, and skeletal muscle mass, the participants were restricted to young adults in order to control the variables and to simplify the study structure. To generalize the results of this study, additional studies of elderly patients who have clinical sarcopenia may be needed. Second, the relatively small sample size might have masked some statistical trends. Accordingly, a larger study is needed to clarify the influence of sarcopenia on respiratory muscle strength. Third, the muscle 
strength measurements were volitional tests. That is, the participants had to perform maximal contractions for the results to be true measures of strength. Although all accuracy recommendations suggested by the American Society of Hand Therapists and the American Thoracic Society/European Respiratory Society statement were followed, muscle strength measurements might have been performed sub-optimally, producing a biased estimate.

In conclusion, respiratory muscle strength assessed by MIP had correlations with skeletal muscle mass, knee extensor strength, and hand grip strength in healthy young adults. In contrast, no associations were found for PEF or MEP. However, additional studies are needed to investigate the feasibility of respiratory muscle strength parameters for evaluating muscle mass and functional outcomes of the elderly with sarcopenia.

\section{CONFLICT OF INTEREST}

No potential conflict of interest relevant to this article was reported.

\section{REFERENCES}

1. Doherty TJ. Invited review: aging and sarcopenia. J Appl Physiol (1985) 2003;95:1717-27.

2. Baumgartner RN, Koehler KM, Gallagher D, Romero L, Heymsfield SB, Ross RR, et al. Epidemiology of sarcopenia among the elderly in New Mexico. Am J Epidemiol 1998;147:755-63.

3. Cruz-Jentoft AJ, Baeyens JP, Bauer JM, Boirie Y, Cederholm T, Landi F, et al. Sarcopenia: European consensus on definition and diagnosis: Report of the European Working Group on Sarcopenia in Older People. Age Ageing 2010;39:412-23.

4. Clark BC, Manini TM. What is dynapenia? Nutrition 2012;28:495-503.

5. Delmonico MJ, Harris TB, Visser M, Park SW, Conroy MB, Velasquez-Mieyer P, et al. Longitudinal study of muscle strength, quality, and adipose tissue infiltration. Am J Clin Nutr 2009;90:1579-85.

6. Bean JF, Kiely DK, Herman S, Leveille SG, Mizer K, Frontera WR, et al. The relationship between leg power and physical performance in mobility-limited older people. J Am Geriatr Soc 2002;50:461-7.

7. Manini TM, Visser M, Won-Park S, Patel KV, Strot- meyer ES, Chen $\mathrm{H}$, et al. Knee extension strength cutpoints for maintaining mobility. J Am Geriatr Soc 2007;55:451-7.

8. Drouin JM, Valovich-mcLeod TC, Shultz SJ, Gansneder BM, Perrin DH. Reliability and validity of the Biodex system 3 pro isokinetic dynamometer velocity, torque and position measurements. Eur J Appl Physiol 2004;91:22-9.

9. Surburg PR, Suomi R, Poppy WK. Validity and reliability of a hand-held dynamometer with two populations. J Orthop Sports Phys Ther 1992;16:229-34.

10. Kollock RO Jr, Onate JA, Van Lunen B. The reliability of portable fixed dynamometry during hip and knee strength assessments. J Athl Train 2010;45:349-56.

11. Keating JL, Matyas TA. The influence of subject and test design on dynamometric measurements of extremity muscles. Phys Ther 1996;76:866-89.

12. Chen LK, Liu LK, Woo J, Assantachai P, Auyeung TW, Bahyah KS, et al. Sarcopenia in Asia: consensus report of the Asian Working Group for Sarcopenia. J Am Med Dir Assoc 2014;15:95-101.

13. Buchman AS, Boyle PA, Wilson RS, Gu L, Bienias JL, Bennett DA. Pulmonary function, muscle strength and mortality in old age. Mech Ageing Dev 2008;129:625-31.

14. Buchman AS, Boyle PA, Wilson RS, Leurgans S, Shah $\mathrm{RC}$, Bennett DA. Respiratory muscle strength predicts decline in mobility in older persons. Neuroepidemiology 2008;31:174-80.

15. Evans JA, Whitelaw WA. The assessment of maximal respiratory mouth pressures in adults. Respir Care 2009;54:1348-59.

16. Syabbalo N. Assessment of respiratory muscle function and strength. Postgrad Med J 1998;74:208-15.

17. Buckinx F, Reginster JY, Dardenne N, Croisiser JL, Kaux JF, Beaudart C, et al. Concordance between muscle mass assessed by bioelectrical impedance analysis and by dual energy X-ray absorptiometry: a cross-sectional study. BMC Musculoskelet Disord 2015;16:60.

18. Janssen I, Heymsfield SB, Baumgartner RN, Ross R. Estimation of skeletal muscle mass by bioelectrical impedance analysis. J Appl Physiol (1985) 2000;89:465-71.

19. Kyle UG, Bosaeus I, De Lorenzo AD, Deurenberg P, Elia M, Manuel Gomez J, et al. Bioelectrical imped- 
ance analysis. Part II: utilization in clinical practice. Clin Nutr 2004;23:1430-53.

20. Janssen I, Heymsfield SB, Ross R. Low relative skeletal muscle mass (sarcopenia) in older persons is associated with functional impairment and physical disability. J Am Geriatr Soc 2002;50:889-96.

21. Pedrero-Chamizo R, Albers U, Tobaruela JL, Melendez A, Castillo MJ, Gonzalez-Gross M. Physical strength is associated with Mini-Mental State Examination scores in Spanish institutionalized elderly. Geriatr Gerontol Int 2013;13:1026-34.

22. Arai H, Akishita M, Chen LK. Growing research on sarcopenia in Asia. Geriatr Gerontol Int 2014;14 Suppl 1:1-7.

23. Innes EV. Handgrip strength testing: a review of the literature. Aust Occup Ther J 1999;46:120-40.

24. Kim WK, Kim DK, Seo KM, Kang SH. Reliability and validity of isometric knee extensor strength test with hand-held dynamometer depending on its fixation: a pilot study. Ann Rehabil Med 2014;38:84-93.

25. Katoh M, Yamasaki H. Comparison of reliability of isometric leg muscle strength measurements made using a hand-held dynamometer with and without a restraining belt. J Phys Ther Sci 2009;21:37-42.

26. Servio TC, Pereira RS, Cataneo DC. Study on functional cardiorespiratory changes after laparoscopic Nissen fundoplication. Acta Cir Bras 2012;27:499-504.

27. Pessoa IM, Coelho CM, Mendes LP, Montemezzo D, Pereira DA, Parreira VF. Comparison of three protocols for measuring the maximal respiratory pressures. Fisioterapia em Movimento 2015;28:31-9.

28. American Thoracic Society; European Respiratory Society. ATS/ERS Statement on respiratory muscle testing. Am J Respir Crit Care Med 2002;166:518-624.

29. Rantanen T, Guralnik JM, Foley D, Masaki K, Leveille S, Curb JD, et al. Midlife hand grip strength as a predictor of old age disability. JAMA 1999;281:558-60.

30. Ploutz-Snyder LL, Manini T, Ploutz-Snyder RJ, Wolf DA. Functionally relevant thresholds of quadriceps femoris strength. J Gerontol A Biol Sci Med Sci 2002;57:B144-52.
31. Newman AB, Kupelian V, Visser M, Simonsick EM, Goodpaster BH, Kritchevsky SB, et al. Strength, but not muscle mass, is associated with mortality in the health, aging and body composition study cohort. J Gerontol A Biol Sci Med Sci 2006;61:72-7.

32. Cesari M, Pedone C, Chiurco D, Cortese L, Conte ME, Scarlata S, et al. Physical performance, sarcopenia and respiratory function in older patients with chronic obstructive pulmonary disease. Age Ageing 2012;41:23741.

33. Greising SM, Mantilla CB, Gorman BA, Ermilov LG, Sieck GC. Diaphragm muscle sarcopenia in aging mice. Exp Gerontol 2013;48:881-7.

34. van der Palen J, Rea TD, Manolio TA, Lumley T, Newman AB, Tracy RP, et al. Respiratory muscle strength and the risk of incident cardiovascular events. Thorax 2004;59:1063-7.

35. Huang CH, Yang GG, Wu YT, Lee CW. Comparison of inspiratory muscle strength training effects between older subjects with and without chronic obstructive pulmonary disease. J Formos Med Assoc 2011;110:518-26.

36. Jain P, Kavuru MS, Emerman CL, Ahmad M. Utility of peak expiratory flow monitoring. Chest 1998;114:86176.

37. Kim J, Davenport P, Sapienza C. Effect of expiratory muscle strength training on elderly cough function. Arch Gerontol Geriatr 2009;48:361-6.

38. Tamplin J, Berlowitz DJ. A systematic review and meta-analysis of the effects of respiratory muscle training on pulmonary function in tetraplegia. Spinal Cord 2014;52:175-80.

39. Simoes LA, Dias JM, Marinho KC, Pinto CL, Britto RR. Relationship between functional capacity assessed by walking test and respiratory and lower limb muscle function in community-dwelling elders. Rev Bras Fisioter 2010;14:24-30.

40. Kim J, Sapienza CM. Implications of expiratory muscle strength training for rehabilitation of the elderly: Tutorial. J Rehabil Res Dev 2005;42:211-24. 